"Durchsichtige Wörter" bestehen aus mindestens zwei Morphemen. Eine Analyse der Bestandteile und die Kenntnis des Wortbildungsprogrammes führen zur vollstädigen Definition des mehrmorphemischen. Wortes. Diese vollkommene Durchsichtigkeit wird eingeschränkt durch

a) Lexikalisierung

b) prinzipielle Teildurchsichtigkeit bestimmter Wortbildungsprogramme

c) Gegebenheiten, die vom Sprachbenutzer abhängig sind.

In einem Vortrag, den ich 1979 in Ljubljana gehalten habe, war das Thema dieses Beitrags bereits angedeutet. Ich untersuchte damals die Frequenzwörterbücher der romanischen Sprachen und stellte die Frage, inwieweit ein Vergleich der Frequenzwörterbücher des Französischen, Italienischen, Rumänischen etwas zur Charakteristik dieser Sprachen beitragen kann. Einer der Punkte, an denen ich die Möglichkeiten eines Vergleichs erprobte, war die von Sprache zu Sprache verschiedene Menge der durchsichtigen Wörter. Schon damals hielt ich allerdings den Terminus durchsichtig für problematisch:

"in der Welt der Dinge sind z.B. Luft, Fensterglas, farbiges Glas, Wasser (mehr oder weniger verschmutzt) durchsichtig, allerdings in jeweils verschiedenem Maße. Auch beim Blattwerk eines Strauches oder Baumes kann ich von Durchsichtigkeit sprechen, jedoch funktioniert hier die Durchsichtigkeit bzw. ihre Beeinträchtigung in anderer Weise als etwa bei verschmutztem Wasser oder Milchglas. Für die Durchsichtigkeit der Wörter ergeben sich ähnliche Probleme"1)

1) G. Ennst: Das Frequenzwörterbuch - ein Hilfsmittel der vergleichenden Sprachcharakteristik? in: G. Ennst /A. Stefenelii. (Hg.), Sprache und Mensch in der Romania. Festschrift Heinrich Kuen zum 80. Geburtstag, Wiesbaden 1979,30 . 
Von den damals nur angedeuteten Problemen der Wortdurchsichtigkeit soll hier ausführlicher die Rede sein.

Ich will als Einstieg in die Problematik mit einer kleinen sprachlichen Beobachtung beginnen, die ich im vergangenen Sommer machen konnte, als ich mit meiner Familie einen Teil der Ferien auf einer Insel vor der dalmatinischen Küste verbrachte. Wie jeder brave Tourist unternahmen auch wir mit dem Bus eine Exkursion entlang der dalmatinischen Küste und nach Mostar. Außer Deutschen saßen auch Engländer im Bus; der Reiseführer gab deshalb seine Erklärungen jeweils auf Deutsch und Englisch. Zu Beginn der Fahrt sagte er zunächst auf Englisch: "I'll give you some basic information", danach auf Deutsch: "Ich werde Ihnen einige gründiche Erklärungen geben". Den Mitfahrern im Bus war weiter nichts aufgefallen, die Außerung war ja auch nicht sehr wichtig. Nur der Sprachwissenschaftler, an seinen Rotstift für Ubersetzungskorrekturen gewöhnt, störte sich an dem Fehler basic = gründzich. Ich behaupte, daß bei der Entstehung dieses. Fehlers die Durchsichtigkeit von Wörtern bzw. deren Einschränkung eine Rolle spielte; auf die Problematik von basie/gründlich werde ich weiter unten zurückkommen.

\section{Vollkommene Durchsichtigkeit}

Mit dem Terminus Durchsichtigkeit (oder auch Transparenz) wird im allgemeinen die morphologische Motiviertheit von Wörtern bezeichnet. Warum heißt der Apfel im Deutschen Apfel, im Französischen pomme? Eine solche Frage ist im Rahmen einer synchronen Sprachbetrachtung sinnlos; hier kann man nur auf das Saussuresche arbitraire du signe verweisen. Weniger sinnlos ist natürlich die Frage: warum heißt der Apfelbaum im Deutschen Apferbaum, im Französischen pommier? Eine etwas simplifizierte Antwort könnte lauten: weil es ein Baum ist, der Äpfel trägt. Oder weil 
-ier ein Suffix zur Bezeichnung von Bäumen und Sträuchern ist; geht dem Suffix -ier der Name einer Frucht voraus, so bezeichnet das ganze Wort denjenigen Baum oder Strauch, der diese Frucht trägt ${ }^{2}$. Für diese Möglichkeit, die Bedeutung mehrmorphemischer Wörter mit Hilfe ihrer Bestandteile und ihrer Bildungsweise zu erfassen, hat sich in der deutschen Sprachwissenschaft der Begriff Durchsichtigkeit (Transparenz) eingebürgert ${ }^{3 \text { ) }}$.

Die genannten wörter dt. Apfelbaum, frz. pommier zeigen vollkommene Durchsichtigkeit. Wer diese wörter noch nie gehört hat, jedoch ihre Bestandteile und das hier vorliegende Wortbildungsverfahren kennt, kann auch ohne Kontext mit hoher Sicherheit auf die Bedeutung des mehrmorphemischen Wortes schließen. Dies ist der Fall, obwohl im Französischen auch zwei andere Suffixe -ier existieren, das eine zur Bildung von Berufsbezeichnungen (ouvrier, hôtezier, jardinier), das andere zur Bildung von Relationsadjektiven (réseau routier, navigation êtière): Homonymie von Einzelwörtern, von Affixen, vielleicht sogar von Wortbildungsprogrammen $^{4}$ ) ändert prinzipiell nichts an der vollkommenen Durchsichtigkeit derartiger wörter. Sie ist dadurch gegeben, daß Apferbaum, pommier sich jeweils in ein Wortbildungsmuster einfügen, das eine ganze Serie ähnlicher wörter

2) Vgl. zu diesem Wortbildung-"Programm" E. Dieckmann, Wortbildungsstruktur und Mengenlehre in $\operatorname{ZrP} 89,1973,52-65$.

3) Bereits W. Wilmanns 1896, M. Wandruszka 1958 (zit. nach Fill, s.u., S. 16), besonders aber M. Gauger mit seinem Buch Durchsichtige Wörter, Heidelberg 1971; zuletzt A. Fill, Wortdurchsichtigkeit im Englischen, Innsbruck 1980; W. Rettig, Sprachliche Motivation. Zeichenrelationen von Lautform und Bedeutung am Beispiel französischer Lexikoneinheiten, Frankfurt 1981.

4) Damit meine ich die Tatsache, daß etwa pommiex theoretisch auch einen Menschen bezeichnen könnte, der berufsmäßig mit Äpfeln zu tun hat. 
(Kirschbaum, Birnbaum ...; cerisier, poirier...) hervorbringt. Unsere Beispielwörter weichen von diesem Programm weder auf der Ausdrucks- noch auf der Inhaltsseite ab. Die Durchsichtigkeit - gegeben durch die Regelhaftigkeit des Wortbildurgsmusters - ist in diesen Fällen so stark, daß man auf den Eintrag des Wortes im Wörterbuch auch verzichten kann. So finden sich etwa in meinem Exemplar des Duden ${ }^{5)}$ zwar Apfelbaum, Birnbaum, Pflaumenbaum, aber nicht Zwetschgenbaum, Quittenbaum. In französischen wörterbüchern könnte man auf Einträge der folgenden Art leicht verzichten: joyeusement "d'une manière joyeuse", objectivement "d'une manière objective", tendencieusement "d'une manière tendencieuse".

Wäre der ganze Bereich der Wortbildung in dieser Weise regelmäßig, mehrmorphemische wörter immer so vollkommen durchsichtig, so wäre dies u.a. eine bedeutende Erleichterung beim Erlernen einer Fremdsprache: an die Stelle einer sehr großen Menge einzeln zu lernender wörter würde eine kleinere Menge von Regeln treten, mit deren Hilfe mehrmorphemische Wörter gebildet bzw. voll verstanden würden. Als Konsequenz würden die Wörterbücher dünner, die Bildungsregeln wären in die Grammatik aufzunehmen. Hierzu würden selbstverständich auch morphonologische Regeln gehören, welche die jeweiligen lautlichen bzw. graphischen Anderungen der beteiligten Morpheme berücksichtigen. Beispiele: [' $\not]+$ $[m \tilde{\alpha}] \rightarrow\left[\phi z(\partial)^{\prime} m \tilde{\alpha}\right]$ (vgl. tendencieux + tendencieusement); $[' \phi]+\left[i^{\prime} t e\right] \rightarrow[o z i ' t e]$ (vgl. nerveux $\rightarrow$ nervosité). Da derartige morphonologische Veränderungen regelhaft sind, wird durch sie die Durchsichtigkeit der mehnmorphemischen Wörter nicht eingeschränkt.

5) Bibliographisches Institut Mannheim, Der Große Duden, Band 1, Mannheim 161967. 
Nun gibt es aber einen sehr großen Bereich des Wortschatzes, der weder vollkommen durchsichtig ist, noch vollkommen undurchsichtig wie etwa Apfel, Mond etc. bzw. pomme, lune. Sicher wurde in der bisherigen sprachwissenschaftlichen Literatur schon öfters von eingeschränkter bzw. teilweiser Durchsichtigkeit gesprochen. In den vorliegenden Abhandlungen wird aber meist nur e i n Aspekt solcher Einschränkungen betrachtet. Der vorliegende Beitrag soll zeigen, daß die Durchsichtigkeit von Einschränkungen ganz verschiedener Art betroffen sein kann.

\section{Trennung des mehrmorphemischen Wortes von seinen Bestand- teilen}

Die hier zu besprechenden Phänomene betreffen jeweils einzelne wörter. Vom historischen Moment an, in dem zwei Morpheme erstmals zusammen als $e i n$ Wort auftreten, unterliegen sowohl das jetzt neu gebildete Wort als auch die dieses Wort konstituierenden Morpheme, soweit sie außerhalb dieses Wortes auftreten, den Zufällen der Geschichte, der sprachlichen Entwicklung. Diese Entwicklung kann zu einer gewissen Distanz zwischen dem mehrmorphemischen Wort und seinen Bestandteilen in selbständiger. Verwendung führen. Der Vorgang wird allgemein als Lexikalisierung bezeichnet. Mögliche Gründe sind: der Untergang wenigstens eines der Bestandteile oder eine auseinanderstrebende lautliche oder semantische Entwicklung. Das Resultat ist jeweils dasselbe: die eindeutige Beziehung wenigstens eines der Bestandteile des mehrmorphemischen Wortes zu einem identischen Morphem in selbständiger Verwendung geht verloren.

1. Schwund einzelner Bestandteile

Lat. Zaetus "froh" hat afz. Zié engeben, laetitia wurde zu Ziesse. Im Lateinischen und im Altfranzösischen sind Zaetitia bzw. liesse vollkommen durchsichtig. Im heutigen 
Französisch existiert nur mehr Ziesse "joie débordante et collective" als ein vorwiegend literarisches wort (un peuple en liesse). Dieses ist aber zumindest in seinem ersten Bestandteil keineswegs mehr durchsichtig, da dieser als selbständiges Wort im Laufe der Jahrhunderte untergegangen ist.

recipere mag im Lateinischen ein voll durchsichtiges wort gewesen sein (re + capere); auf dem Weg zu frz. recevoir. hat es seine Durchsichtigkeit verloren, da lat. capere sich nicht ins Französische hinein fortgesetzt hat ${ }^{6)}$.

Für frz. prélude kann ich zwar Vermutungen über die Bedeutung bzw. Funktion von pré-haben, diese führen aber nicht weit, da * Zude kein Morphem der frz. Sprache ist ${ }^{7}$ ).

2. Lautliche Trennung

Das mehrmorphemische Wort und seine Bestandteile in selbständiger Verwendung können jeweils die erbwöntliche Entwicklung durchlaufen; eine evtl. später daraus resultierende lautliche Trennung ist für das synchrone Sprachbewußtsein dennoch in vielen Fällen relativ leicht überbrückbar, denn die Verschiedenheiten der lautlichen Entwicklung führen zu Entsprechungsregeln in der Synchronie. Vgl, etwa die Entwicklung von lat. á[ und à [ :

Diachronische Regeln: 1. á $[\rightarrow[\varepsilon]$

$$
\text { 2. } \mathrm{a}[\rightarrow[a]
$$

Synchronische Entsprechungsregel: betontes $[\varepsilon] \approx$ nebentoniges [a].

Diesen Regeln entsprechen etwa mer / marin, clair / clarté.

6) Der Versuch, ein Morphem *cevoir aus recevoir, concevoix, apercevoir zu erschließen, führt wohl nicht weit.

7) Daß prélude ein Latinismus ist, spielt an dieser stelle keine Rolle. 
Weitere synchronische Entsprechungsregeln dieser Art im Französischen:

$[\tilde{\varepsilon}]$ unter dem Hauptton $\approx[a]$ oder $[\tilde{\alpha}]$ unter dem Nebenton: sain / santé, main / manette ${ }^{8)}$; $[œ, \emptyset]$ unter dem Hauptton $\approx$ [u] unter dem Nebenton: jeu / jouer, neuf / nouveau ${ }^{8)}$.

In den hier genannten Fällen beeinträchtigt die Lautentwicklung wohl noch nicht die Durchsichtigkeit des abgeleiteten Wortes. Inwiefern solche Entsprechungen vom durchschnittlichen Sprachbenutzer als regelhaft empfunden werden, hängt allerdings weitgehend von der Anzahl ähnlicher Fälle ab.

Darüber hinaus haben win gerade im Französischen häufig den Fall, daß ein Wort alle Stufen der Lautentwicklung mitgemacht hat, während für die hiervon $z u$ erwartende $A b-$ leitung in irgendeinem stadium der frz. Sprachgeschichte auf das entsprechende lat. Wort zurückgegriffen wurde. Der Latinismus wurde nur teilweise den inzwischen erfolgten Lautveränderungen unterworfen. So kommt es, daß besonders im Französischen häufig das Grundwort und die jedenfalls semantisch dazugehörige "Ableitung" lautlich so weit voneinander entfert sind, daß man von einer Durchsichtigkeit nicht mehr sprechen kann: mür / maturité, frère / fraternel,

8) Von der Frage der Regelmäßigkeit bei der s e m a n $t i s c h e n$ Entsprechung ist hier zunächst einmal abgesehen. 
(ré)soudre/ solution sind oft genannte Beispiele für dieses Phänomen ${ }^{9)}$.

\section{Semantische Trennung}

Wenn die Dinge und Verhältnisse in der Realität sich ändern, das zunächst durchsichtige Wort aber bleibt, so hat dies Konsequenzen für seine Durchsichtigkeit. Dt. B.leistift hat wesentliche Elemente seiner Durchsichtigkeit eingebüßt, seitdem ein solcher stift nicht mehr mit Blei, sondern mit Graphit hergestellt wird. Frz. Zuthier (seit 1649) war zunächst sicher durchsichtig: der damit bezeichnete Handwerker stellte hauptsächlich Lauten her. Als die Verwendung der Laute in der Musik zurücktrat, spezialisierten sich die Handwerker auf die nun moderneren Streichinstrumente, so daß ein heutiger Zuthier "Geigenbauer" evtl. noch nie eine Laute gebaut hat. Die Analyse einer solchen Bildung erkennt zwar zwei Morpheme, sie würde aber zu einem falschen Resultat hinsichtlich der Bedeutung des Gesamtwortes kommen.

Natürlich sind Bedeutungsveränderungen des abgeleiteten Wortes, semantische Abweichungen vom jeweiligen Programm, Spezialisierungen im Einzelfall auch ohne Veränderungen. der jeweils bezeichneten Realität möglich.

Das Programm der frz. Adverbbildung erscheint - von Randphänomenen abgesehen - morphologisch und in den jeweiligen semantischen Beziehungen besonders regelmäßig. Dennoch findet man ohne große Mühe eine Reihe von Fälien, wo das Adverb nicht mit "d'une façon + adj." bzw. "avec + subst. de

9) $\mathrm{Zu}$ Versuchen, die verlorengegangene Durchsichtigkeit wieder herzustellen, vgl. A. Stefenelli: Remotivationstendenzen in der Geschichte des französischen Wortschatzes, in: G. Ennst/A. Stefenelli (Hg.) (vgl. Anm. 1), 179-192. 
qualité" definiert werden kann ${ }^{10}$ ). Ich vermute, daß es sich hier in den meisten Fällen um Resultate historischer Entwicklungen handelt, nicht um ein Abweichen vom Programm beim ersten Auftreten:

Paul dort tranquilzement f "d'une façon tranquille", sondern "en tranquilité"11).

Pierre dort certainement * "d'une façon certaine", sondern: "je suis sur (certain) que Pierre dort"12)

Hierher gehören die ganzen Satzadverbien oder Modalisatoren, wie heureusement, évidemment, naturellement etc. ${ }^{13)}$, von denen ich vermute, daß sie unsprünglich nur das Verb modifizierten; ihre Verwendung mit Bezug auf den Satz bzw. auf die Bewertung der Aussage kam wohl meist erst später dazu und verdrängte in vielen Fällen die ursprüngliche Bedeutung bzw. Funktion. Bei Adverbien wie nouverzement und récemment ist die vom semantischen Programm ("d'une manière, façon + Adj.") abweichende Bedeutung vielleicht von Anfang an allein vorhanden gewesen. Bei rondement und carrément stellen wir fest, daß die zugrundeliegenden Adjektive einen gewissen Gegensatz bilden, die Adverbien aber in Ausdrücken wie dire carrément (rondement) son opinion synonym verwendet werden können; hier zeigt sich eine semantische Entwicklung des abgeleiteten Wortes, die in extremer Weise vom zugrundeliegenden lexematischen Bestandteil wegführt.

10) Zur Typologie der frz. Adverbien vgl. Chr. Schwarz: Der nicht-nominale ment-Ausdruck im Französischen, München 1980 .

11) Seit 17. Jh. (FEW 13,2,197a).

12) In dieser Verwendung wohl erst seit dem 16. Jh.; vgl. FEW 2,1,611a.

13) Vgl. G. Ernst: Adverb und ModaZisator im Französischen, in: ZFSL $87,1977,1-19$. 
Derartige semantische Entwicklungen (oder Abweichungen im Moment der Neubildung) machen natürlich eine Aufnahme des. abgeleiteten Wortes ins Wörterbuch notwendig (im Gegensatz zu den obengenannten regelmäßigen Fällen wie joyeusement, objectivement, tendencieusement etc.). Als "Lexikalisierung" wollen wir aber nur die semantische Entwicklung des abgeleiteten (mehrmorphemischen)Wortes verstehen, die zu einer Abweichung von den zugrundeliegenden Bestandteilen und/oder vom Programm führt; die Aufnahme ins Wörterbuch, ins Lexikon ist lediglich die notwendige Folge dieses Vorgangs.

An Beispielen aus dem Bereich der frz. substantifs de qualité soll nun der Vorgang der semantisch bedingten Lexikalisierung näher betrachtet werden. Ihnen liegt jeweils ein Adjektiv zugrunde, das unter Beibehaltung seiner Semantik in die Klasse der substantive "verschoben" wird ${ }^{14)}$. Beispiele: triste $\rightarrow$ tristesse, riche $\rightarrow$ richesse, pauvre $\rightarrow$ pauvreté, beau $\rightarrow$ beauté, Zaid $\rightarrow$ laideur. Typische Definitionen des wörterbuchs ${ }^{15}$ ) sind hier etwa "caractère de ce qui est beau", "caractère, état de ce qui est laid". Daß diese wörter ins wörterbuch aufgenommen werden müssen, ist wegen der Vielfalt der hier konkurrierenden Wortbildungsprogramme selbstverständich ${ }^{16)}$. Nicht diese Notwendigkeit aber wollen wir als Lexikalisierung bezeichnen, sondern die Tatsache, daß der Bedeutungsumfang des abgeleiteten Wortes denjenigen des Grundwortes bald unter-, bald überschreitet, oder daß die Bedeutung des abgeleiteten Wortes

14) Terminologie nach Gauger (vgl.Anm. 3).

15) Ich übernehme hier in wesentlichen Definitionen und Beispiele des Petit Robert.

16) -eur, -ie, -(i)té, -itude, -ise, -isme, -ice, -ence/-ance, -sion/ -tion .... Natürlich könnte man bei jedem in Frage kommenden Adj. angeben, in welches Ableitungsprogramm der substantifs de qualité es eintreten kann, und dann das Subst.nicht mehr als eigenen. Eintrag führen; dies würde aber prinzipiell nicht Wesentliches änderm. 
sich von der des Grundwortes gänzlich löst; hierbei ist wichtig, daß die Bedeutungsveränderung nicht eine ganze Gruppe, sondern jeweils den Einzelfall betrifft.

vieux: il est vieux $\rightarrow$ je respecte sa vieillesse

jeune: il est trop jeune $\rightarrow$ il a trop de jeunesse (Conneille)

Hier liegt jeweils die semantische Regelmäßigkeit der substantifs de qualité vor: "Ia qualité, le fait d'être jeune (vieux)". In beiden Fällen bezeichnet das substantiv aber auch einen bestimnten Lebensabschnitt (un enfant qui serait le soutien de notre vieillesse, Lautréamont; au temps de ma jeunesse folle, Villon), sowie die Gesamtheit der Personen, die sich in diesem Lebensabschnitt befinden (instruire la jeunesse; aide à la vieizlesse). tendresse ist nicht nur die durch das Adjektiv tendre bezeichnete Eigenschaft, das Gefühl, sondern - wenigstens im Plural - auch der Ausdruck dieses Gefühls: milze tendresses (am Ende eines Briefes).

richesse ist nicht nur die "qualité de ce (celui) qui est riche", sondern auch das konkrete Besitztum, das die Grundlage dieser Eigenschaft bildet.

beauté bezeichnet zunächst den "caractère de ce qui est beau" bzw. die "qualité d'une personne belie", darüber hinaus aber auch eine konkrete Person, für welche diese Eigenschaft charakteristisch ist: épouser une beauté; im Falle von jeunesse existient dieser Gebrauch nur im français familier, ist aber auch dort "vieilli ou régional": épouser une jeunesse; entsprechende Verwendungsweisen von z.B. vieizlesse, richesse wären zwar theoretisch denkbar, sind aber in der konkreten Sprachnorm nicht erlaubt: *épouser une vieizlesse, une richesse.

Reduktionen des Bedeutungsumfangs beim abgeleiteten Wort kommen oft dadurch zustande, daß der Ableitung nur eine 
von mehreren Bedeutungen des Grundwortes zugrundeliegt ${ }^{17}$ ). So lassen sich etwa die verschiedenen Bedeutungen von tendre im wesentlichen um den physischen Aspekt und um den charakterlich-moralischen gruppieren: des haricots verts très tendres, de za viande tendre / une tendre épouse. tendresse bezieht sich aber nur auf die zweite Bedeutungsgruppe, also nur la tendresse d'une femme oder la tendresse que j'éprouve pour une femme, aber nicht *la tendresse des haricots verts; hierfür steht tendreté zur Verfügung.

Ähnliches gilt für bassesse in seiner Beziehung zu bas, Kontexte wie un mur bas, la marée basse, les nuages sont bas, $\grave{a}$ bas prix liefern keine Wortbildungen der Art * Za bassesse du mur etc. bassesse bezieht sich nur auf die Niedrigkeit eines Charakters und einer daraus resultierenden Handlungsweise: La bassesse d'un flatteur, rougir d'une bassesse. Wenn nun unter den verschiedenen Bedeutungen eines Grundwortes gerade diejenige außer Gebrauch kommt, die dem abgeleiteten Wort zugrundeliegt, so führt dies zu einer noch stärkeren. "Entfremdung", Lexikalisierung und damit Minderung der Durchsichtigkeit bei diesem abgeleiteten Wort. gros hat die Bedeutungen "dick" (Sachen und Personen) und "schwanger"; letztere ist aber veraltet, das normale wort ist enceinte. Die Ableitung grossesse bezeichnet jedoch nur die "Schwangerschaft", nicht die "Dicke" (hierfür grosseur). Sollte nun gros(se) "schwanger" ganz ungebräuchlich werden, so hätten wir nebeneinander nur noch enceinte "schwanger" / grossesse "Schwangerschaft"; die Beziehung von grossesse zu gros wäre damit nur noch sehr indirekt, die Durchsichtigkeit in hohem Maß getrübt.

17) F.J. Hausmann (Was ist und was solz ein Lernwörterbuch?, in: ZFSL 84,1974, 97-129, hien S. 116 f.) spricht von Distributionsbeschränkungen der Ableitungen und gibt hierfür die Beispiele vertement, déchirer, épreuve. 
Bedeutungseinschränkungen bzw. -präzisierungen aus dem Bereich der frz. Diminutiva: Livret als "petit Iivre" gilt heute als veraltet; es hat sich auf verschiedene Typen von kleinen "Büchern" spezialisiert: livrét militaire, livret de santé, livret de famille, livret scolaire, livret de caisse d'épargne, livret d'un opéra. Es ist kaum noch möglich, ein beliebiges kleines Buch als livret zu bezeichnen. Vgl. auch noix "Walnuß" / noisette "HaselnuB"; fourche "Gabel als Arbeitsgerät in der Landwirtschaft" / fourchette "Eßgabel"; fosse "Grube" / fossette "Grübchen" (hier hat im Deutschen dieselbe Lexikalisierung stattgefunden wie im Französischen).

Bei gewissen Typen der Wortzusammensetzung, insbesondere der Verbindung $N+$ Adj. im Französischen, muß man sich fragen, ob die Lexikalisierung nicht gerade eine Voraussetzung für den Status eines eigenen Wortes ist. Niemand wird auf die Idee kommen, (une)robe blanche als zusammengesetztes Wort anzusehen; für fromage blanc "Quark" wird man das aber mit einigem Recht tun. Warum? Weil blane hier nicht mehr in seiner üblichen Bedeutung verwendet wird, mit der es in freien syntagmen auftritt. Vgl. auch ferblanc "Blech", chaise Zongue, bon mot etc. Für diesen Typ der Wortzusammensetzung (ich lasse offen, ob dies auch für andere Typen der Fall ist) gilt geradezu: dort, wo eine Verbindung $N+$ Adj. oder Adj. + N voll durchsichtig ist, handelt es sich um kein zusammengesetztes Wort; und umgekehrt: eine derartige Wortzusammensetzung ist immer nur zum Teil durchsichtig. Dies liegt an der in jedem Einzelfall wieder anderen, nicht voraus zu berechnenden Lexikalisierung wenigstens eines der beiden Bestandteile.

Prinzipielle Teildurchsichtigkeit von Wortbildungsmustern Hier handelt es sich - im Gegensatz zum vorigen Abschnitt um Verfahren der Wortbildung, wo die beteiligten Morpheme innerhalb und außerhalb des mehrmorphemischen Wortes mit 
sich selbst identisch sind, d.h. keine semantischen Unterschiede aufweisen, und wo dennoch eine Analyse nicht vollkommen bis zur Bedeutung des mehrmorphemischen Wortes führen kann. Betrachten wir zunächst einige. Beispiele aus dem Deutschen und aus dem Französischen. Die ersten Beispiele entnehme ich Gaugers Buch zu den durchsichtigen wörtern ${ }^{18}$ ): Butterbrot : die Butter ist a u f dem Brot Butterkeks: die Butter ist $i \mathrm{~m}$ Keks Buttermizch: die Butter ist $\mathrm{n} i \mathrm{ch} t \mathrm{meh} \mathrm{r}$ in der"Milch" Butterblume: die Blume $g I \ddot{a} \mathrm{n} z t w i e$ Butter Als mir im Deutschen zum ersten Mal das Wort Denkpause begegnete, war ich zunächst etwas perplex: sollte das eine Pause vom Denken sein, ebenso wie in der Arbeitspause nicht gearbeitet wird? Der Kontext legte schließlich die zutreffende Interpretation nahe: "Pause, die zum Denken genützt werden soll".

Die Wörter Lebensgefahr und Todesgefahr sind zwar nicht unbedingt in allen Kontexten gegeneinander austauschbar; dennoch sind sie halbwegs synonym, obwohl die jeweiligen ersten Bestandteile Gegensätze sind.

Deutsche Studenten des Französischen analysieren das Wort après-ski als "ce qu'on fait après avoir fait du ski"; sie sind dabei beeinflußt von der Tatsache, daß Après-ski mit dieser Bedeutung im Deutschen verwendet wird; selbstverständlich spielen aber auch die parallelen Wortbildungen après-midi, après-guerre eine Rolle; die tatsächliche Bedeutung von après-ski ist aber "bóttilion souple, chaud, qu'on chausse lorsqu'on ne skie pas". Diese Bedeutung gilt übrigens auch für ital. doposei; dopolavoro bezeichnet dagegen keine Schuhe, die man nach der Arbeit trägt, sondern die (organisierten) Aktivitäten in der Freizeit.

18) Gauger 149. 
Noch ein letztes Beispiel, das einem anderen Wortbildungsmuster entnomnen ist: amuse-gueule ist nach einem im Französischen sehr geläufigen Muster $N(V+N)$ gebildet, ähnlich wie pare-brise, coupe-papier, porte-cigarettes. Die Analyse aufgrund der Bestandteile und des Wortbildungsprogrammes wird so etwas ergeben wie "qch (ou qn?) qui amuse la gueule"; weiter kann die Analyse nicht führen, und wer das wort nicht bereits als Ganzes kennt, wird kaum auf die im Petit Robert gegebene Bedeutung kommen: "Petit sandwich, biscuit salé etc., servi avec l'apéritif".

Ich breche hier die Reihe der Beispiele ab. Im Gegensatz zum vorigen Abschnitt kann man hier nicht von Lexikalisierung in der Weise sprechen, daß eines der beteiligten Morpheme innerhalb und außerhalb der Zusammensetzung jeweils eine andere Bedeutung hätte: in einer Definition "chaussures qu'on met après avoir fait du ski" haben après und $s k i$ jeweils dieselbe Bedeutung wie in après-ski. Das Entscheidende aber ist, $\mathrm{d} a ß$ wesentliche Teile dieser Definition nicht aus den Einzelelementen und aus der Kenntnis des Wortbildungsverfahrens zu erschließen sind. In unserem Fall wäre dies das Element "chaussures qu'on met ..."; im Fall der oben gebrachten deutschen Beispiele mit Buttersind dies die Definitionselemente "auf", "in", "nicht mehr in", "(glänzt) wie". Dies ist ein grundlegendes und wohlbekanntes Problem der gesamten Wortbildungslehre, das insbesondere in den Arbeiten zur Komposition, zur Wortzusammensetzung eine große Rolle spielt. Beim Versuch, derartige Wortbildungen systematisch zu erfassen, scheinen mir drei wissenschaftliche Positionen denkbar:

1. Resignation gegenüber der Vielfalt denkbarer Beziehungen zwischen den Elementen. Dies könnte dazu führen, daß man etwa im Deutschen Wortbildungen wie Butterbrot, also den Typ $\left.N_{\left(N_{1}\right.}+N_{2}\right)$ nur analysiert als "ein $N_{2}$, das in ir- 
gendeiner Beziehung zu $N_{1}$ steht".

2. Man kann auch versuchen, die Zahl der möglichen Beziehungen einzugrenzen, indem man eine endliche zahl recht allgemeiner Typen von Beziehungen aufstellt. Ähnlich wie zwischen den Elementen eines Satzes nur eine e $\mathrm{n} d \mathrm{l} c \mathrm{~h}$ e ZahI von Beziehungen denkbar ist (so etwa in der Kasusgrammatik), so könnte dies ja auch für Wortbildungen, insbesondere Wortzusammensetzungen gelten. Unter verschiedenen Versuchen in dieser Richtung gehe ich hier kurz auf denjenigen der amerikanischen Autorin Judith N. Levi ein ${ }^{19)}$. Levi kommt im Fall der Nominalkomposita auf 12 denkbare Beziehungen, die sie zwar anhand englischer Beispiele gewornen hat, denen sie aber offenbar den Charakter von Universalien zusprechen möchte. Ein Nominalkompositum sei dann prinzipiell von 12 facher Ambiguität. Eine derart vielfache Ambiguität bzw. Homonymie/Polysemie mus nicht unbedingt gegen Levis Theorie sprechen. $\mathrm{Zu}$ prüfen wäre allenfalls, ob - unter Beschränkung auf die Nominalkomposita - die Zahl von 12 Beziehungen ausreichend ist, $o b$ es gerade diese Beziehungen sind und - wenn $j a$ - wie weit man diese Typen von Beziehungen über das Englische hinaus auf andere Sprachen verallgemeinern darf. Selbst wenn man hierauf immer positiv antworten könnte, so sind damit Probleme jenseits des Bereiches der Nominalkomposita noch nicht gelöst. Inbesondere in Fällen wie après-ski, amuse-gueuze wird man über eine sehr allgemeine Analyse wie "quelque chose qui vient après avoir fait du ski", "quelque chose qui amuse la gueule" kaum hinauskommen. Die Schwierigkeit liegt in der Präzisierung des "quelque chose"; ob dies nun bei amuse-gueule die Lippen einer Frau, ein Slivovitz oder die zum Aperitif gereichten Kekse sind - dieser Frage kann man linguistisch kaum beikommen.

19) Judith N. Levi: The Syntax and Semantics of Complex Nominals, New York 1978. 
3. Mit Bezug auf Nominalkomposita mag man sich fragen: muß denn wirklich für jede derartige Wortbildung mit $x$-facher (z.B. nach Levi 12facher) Ambiguität gerechnet werden? Haben nicht auch Substantive so etwas wie Valenzen, die eine oder mehrere Arten von Beziehung ermöglichen, andere Arten ausschließen? über ein Filtèr etwa läßt sich sagen:
a) Der Filter besteht aus ...
b) Der Filter filtert ...
c) Der Filter hält zurück ...
d) Der Filter dient zur Herstellung von ...
e) Der Filter befindet sich an ...

Vielleicht habe ich denkbare Typen von Aussagen über Filter vergessen. Ihre Anzahl ist jedenfalls nicht unendlich, vielleicht auch geringer als 12; sie ist aber abhängig von der Bedeutung des Wortes Filter und verschieden von Anzahl - und Art - der Aussagen etwa uber ein Buch. Eine ähnliche Typologie ließe sich auch aufstellen für mögliche Determinanten zu Filter. Die Vereinbarkeit der beiden Typologien wïrde dann jeweils zur Zuordnung einer bestimmten Wortbildung zu einem der vorgegebenen Typen führen: Papierfilter, Kohlefilter (a), Wasserfilter, Luft filter (b), Schmutzfilter, Rotfilter (c), Kaffeefilter, Teefilter (d), Zigarettenfilter (e). Arbeiten in dieser Richtung zu Neubildungen im Bereich der deutschen Nominalkomposita werden gegenwärtig in Regensburg unter der Leitung meines Kollegen Brekle durchgefuhrt 20). Ich will den Ergebnissen derartiger Untersuchungen nicht vorgreifen und will hier am Rande lediglich auf zwei Dinge hinweisen.

a) Die Einschränkung der Durchsichtigkeit, wie sie in après$s k i$, comse-gueule oder Buttermilch vorliegt, - ist prinzipiell von der oben genannten Lexikalisierung verschieden. Im Falle der Lexikalisierung ist in Einzelfällen werigstens einer der zwei oder mehr Bestandteile nicht mehr identisch mit einem entsprechenden Morphem im freien Syntagma. Die

20) Für Informationen und Gespräche danke ich Herrn Brekle und seiner Mitarbeiterin J. Boas-Beier. 
Verschiedenheiten können lautlicher und/oder semantischer Art sein. Bei der prinzipiellen Teildurchsichtigkeit mancher Wortbildungsmuster ist zwar die Identität der Morpheme innerhalb und außerhalb des mehrmorphemischen Wortes gegeben. Dennoch funktionieren diese Wortbildungsmuster so, daß eine Analyse von vornherein nur bis zu einer gewissen Stufe vordringen kann; eine präzise Definition des so gebildeten Wortes würde die Entscheidung zwischen mehreren oder sogar unendlich vielen Möglichkeiten der Beziehung zwischen dem Ganzen und seinen Elementen erfordern. Für diese Entscheidung gibt das jeweilige Wortbildungsmuster aber keine Hilfe.

Prinzipielle Teildurchsichtigkeit und Lexikalisierung können zwar in konkreten Einzelfällen gemeinsam vorhanden sein, sie müssen aber dennoch auseinandergehalten werden und dürfen wegen ihrer Verschiedenartigkeit nicht einfach addiert werden. Ich greife wieder auf ein Beispiel Gaugers zurück, das deutsche Wort Briefmarke. Gauger analysiert diese Bildung als "Marke, die für Briefe verwendet wird"; er fährt dann fort:

"Es gibt verschiedene Arten von Marken: Rabattmarken, Gebührenmarken, Versicherungsmarken; eine davon ist die Briefmarke. Mehr läßt sich aus den Gliederinhalten nicht herausholen. Nun wird man eine solche Beschreibung des Inhalts von Briefmarke zwar nicht geradezu als falsch, aber doch als in hohem Maße unvollständig ansehen müssen. Zunächst fehlt die entscheidende Angabe des Zwecks dieser Marke (Beförderung durch die Post), sodann wird, wie jedermann weiß, die B r i e fmarke auch für Postkarten und Päckchen verwendet" 21) Was hier von Gauger mit "zunächst" und "sodann" addiert wird, sind im vorliegenden Fall die prinzipielle Teildurchsichtigkeit ("Marke im Zusammenhang mit Briefen", aber welcher Zusammenhang?) und Lexikalisierung (nicht nur Briefe, auch Karten und Päckchen).

21) Gauger 148 . 
b) Ferner ist darauf hinzuweisen, daß zwar das Phänomen der prinzipielien Teildurchsichtigkeit bei Wortzusammensetzungen offenbar am weitesten verbreitet ist; es fehlt aber auch nicht im Bereich der Wortderivation mit Affixen. So ist unser am Anfang genannter Reiseführer; der basic und gründlich gleichsetzte, den Täuschungen der Teildurchsichtigkeit bei suffigierungen erlegen. Wie ist dieser Fehler zustandegekommen? Die Durchsichtigkeit des englischen Adjektivs erlaubt einen Blick auf das Substantiv base "Grund, Grundlage", die morphologische Interpretation von basic führt zu einer semantischen Interpretation "mit Bezug auf die Grundlage". Ähnliches gilt fün dt. gründzich, in dem wir ja auch das lexikalische Element Grund erkennen. Unser Reiseführer hat nun von der etwa identischen Motivation "mit Bezug auf die Grundlage" auf die Bedeutungsgleichheit von basic und gründlich geschlossen. Er hat dabei übersehen, daß der "Bezug zur Grundlage" hier jeweils verschiedener Art ist: das Syntagma basic information läßt an den Aufbau eines elementaren Wissens denken, wobei die spezielleren Details fehlen können; gründliche Erklärungen sind dagegen Erklärungen, die nicht nur an der Oberfläche, an unwesentlichen Details hängenbleiben, sondern auch den Dingen "auf den Grund gehen". In der folgenden Zeichnung habe ich versucht, die verschiedene Art der Beziehung sichtbar zu machen; diese ist weder bei basic noch bei grindlich allein aufgrund der morphologischen Analyse zu erschließen.
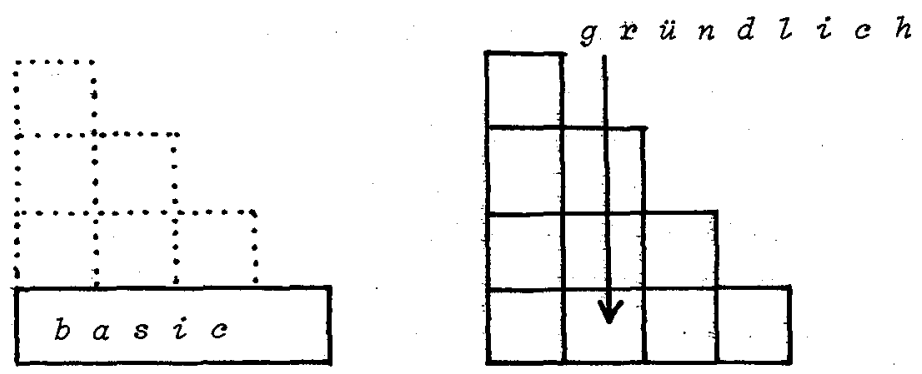
Ein offenbar nur teildurchsichtiges Programm aus dem Bereich der frz. Wortbildung mit Suffixen ist. das folgende: $N\left(N_{1}+-i s t e\right)$.Es dient v.a.zun Bezeichnung von Berufen, wobei das voranstehende lexikalische Element den Gegenstand bezeichnet, der die Grundlage des Berufes bildet. So erscheint uns etwa joumaliste auf den ersten Blick als durchsichtig. Man vergleiche hierzu die Definition des Petit Robert: "Personne qui collabore à la rédaction d'un journal". Woher stammen aber in dieșer Definition die Elemente collabore und rédaction? Sie haben nichts zu tun mit den Bedeutungen von jourmal und -iste, auch nichts mit unseren wissen um das Wortbildungsprogramm: $\mathrm{N}\left(\mathrm{N}_{1}+-i\right.$ ste); sie beruhen nur auf unserer Kenntnis der heute übichen Gesamtbedeutung von journaliste. Auch für den Herausgeber einer Zeitung stellt diese die Grundlage seiner Berufstätigkeit dar; tatsächlich verzeichnet-der Petit Robert als veraltete Bedeutung von journatiste: "Celui qui fait, qui publie un journal". Prinzipiell wäre journaliste aber auch als Bezeichnung für den. Zeitungsverkäufer möglich, ähnilich wie bouquiniste "marchand de livres d!occasion:..": Offenbar ist dem Wortbildungsprogramm nicht zu entnehmen, in welcher der zahlreichen denkbaren Möglichkeiten das journal die Grundlage des Berufes bildet.

Die folgenden Beispiele mögen die Vielfalt denkbarer Beziehungen zum Berufsgegenstand zeigen:

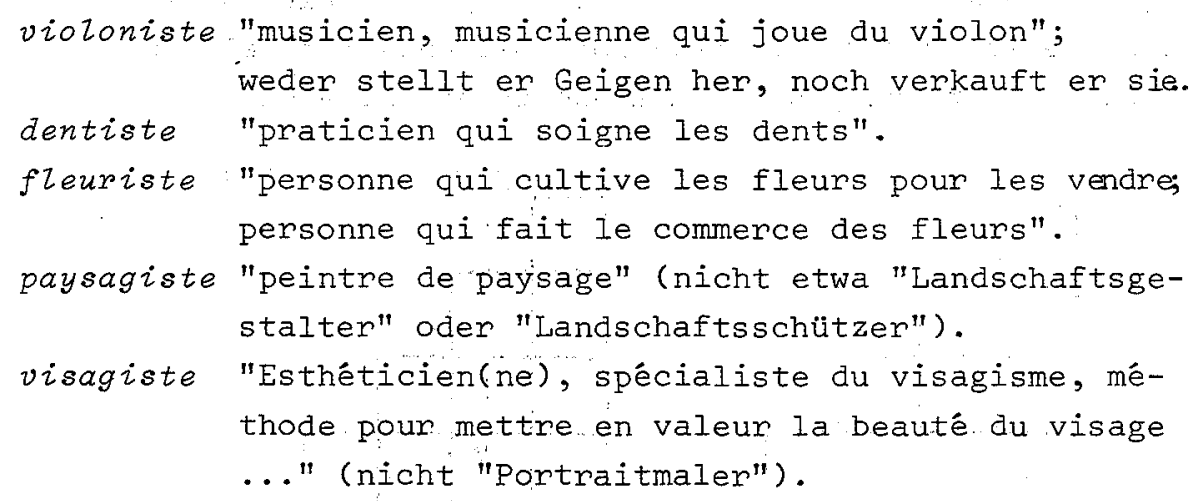


Wer hier die Zahl der denkbaren Beziehungen für einigermaßen begrenzt hält, wird von mehrfacher Ambiguität des Wortbildungsprogramms sprechen müssen; ich selbst tendiere zur Annahme, daß die Zahl der denkbaren Beziehungen nicht angebbar ist: derartige Bildungen auf -iste sind im Französischen prinzipiell nur teilweise durchsichtig.

Eine lohnende Aufgabe jeder einzelsprachlichen Wortbildungslehre dürfte es sein, festzustellen, welche der vorhandenen Wortbildungsprogramme (in Derivation und Komposition) von diesem Phänomen der Teildurchsichtigkeit betroffen sind und welche nicht.

\section{Die Durchsichtigkeit ist abhängig vom Sprachbenutzer}

Viele Dinge des täglichen Gebrauchs sind durchsichtig, ohne daß ich durch sie auch immer hindurchsehe. Die Fenster eines Hörsaals etwa sind durchsichtig, aber nicht in erster linie, damit man durch sie hindurchsieht, sondern damit das Licht einfallen kann. Weingläser, Biergläser sind gewöhnlich durchsichtig; ich benutze sie aber nicht in erster Linie, um durch sie hindurchzusehen. Derartige Feststellungen lassen sich auf die Durchsichtigkeit von Wörtern (sei sie nun vollkommen, durch Lexikalisierung eingeschränkt oder prinzipiell nur teilweise vorhanden) übertragen. Gewöhnlich benutzen wir die wörter, ohne an ihre eventuell vorhandene Durchsichtigkeit auch nur zu denken oder sie wahrzunehmen. Sie wird uns erst bei der Reflexion auf Sprache bewußt. Derartige Reflexion auf Sprache ist Aufgabe des Sprachwissenschaftlers, sie enfolgt aber auch beim Erwerb, bein Erlernen einer Sprache (als Muttersprache oder als Fremdsprache). Im Grunde hat bereits Gauger die Durchsichtigkeit eher als etwas potentiell Vorhandenes dargestellt, als Möglichkeit des Hindurchsehens: "Durchsichtigkeit, is $t$ nicht, sie $g$ e $s h i e h t$, und zwar insofern sie von den Sprechenden 
vollzogen wird"22). Eine neuere Arbeit von W. Rettig entwickelt diesen Gedanken weiter und legt den Akzent vor allem auf die Motivierbarkeit von Wörtern: "Der potentielle Charakter der Motivierbarkeit setzt voraus, dab die blockverfügbaren Einheiten grundsätzlich selbständig nebeneinander zur Sprachkenntnis der Sprecher gehören können" ${ }^{23)}$. Daraus folgt: Durchsichtigkeit spielt eine Rolle beim Sprache $r$ w e $r$ b (gesteuert und ungesteuert), sie ist auch mit Sicherheit ein bedenkenswerter Faktor bei der Sprach b e s c h r e i b u n g, sie spielt aber nur eine sehr untergeordnete Rolle beider alltäglichen Sprach $v$ e $r$ w e n d u $\mathrm{n}$ g. Wir benutzen die Wörter in gleicher Weise, $o b$ wir nun durch sie hindurchsehen (können) oder nicht. Jeder von uns hat wohl schon einmal die folgende Erfahrung gemacht: man verwendet ein Wort (z.B. Augenblick, Grübchen) lange Jahre hindurch vollkommen korrekt und merkt erst später zufällig - daß die Bestandteile des Wortes und seine Bildungsweise einen Blick auf seine Bedeutung freigeben. Da nun aber die Aktualisierung der Durchsichtigkeit vom Sprach benutzer abhängig ist, ist sie auch abhängig von dessen Anstrengung, seiner sprachlichen Vorbildung, seinen intellektuellen Fähigkeiten. Dieser durch Anstrengung, Vorbildung und Fähigkeiten des Sprachbenutzers gegebenen Einschränkung unterliegt alles, was hier bisher gesagt wurde. Das subjekt des Hindurchsehens war bis jetzt ein idealisierter Sprachbenutzer - so weit idealisiert, daß en nicht nur das Sprachsystem korrekt verwendet, sondern auch hierüber richtig reflektiert, insbesondere sich der morphologischen Mehrgliedrigkeit vieler Wörter und der Regeln ihrer Bildungsweise

22) Gauger 12 .

23) Rettig (vgl. Anm. 3) 156. 
bewußt ist ${ }^{24)}$. Wir wissen, daß es diesen idealisierten Sprecher und Sprachreflektierer nicht gibt. Stattdessen gibt es unter diesem Aspekt eine ganze Skala verschieden starker Annäherungen an dieses Ideal. So ist es etwa für einen Franzosen nichts Unnatürliches, die Bildungselemente gelehrter Wörter in seiner Sprache, seinem Idiolekt, verfügbar zu haben - sei es aufgrund seiner Kenntnis des Lateinischen oder Griechischen oder wegen seiner Vertrautheit mit bestimmten Fachsprachen; auch dies gehört ja zur "Mehrsprachigkeit des Menschen"25). Solchen Sprachbenutzern sind vielleicht die Bildungselemente in carnivore, ovipare, oviforme, ovö̈de, ovipositeur, ignifuge, ignivome vertraut und damit die jeweiligen wörter durchsichtig; andere kennen vielleicht nur einige dieser Bildungselemente, jeder etwas anderes. Ich selbst hatte den Vorzug, auf einem Humanistischen Gymnasium Griechisch zu lemen; das erleichtert es mir, die Rechnungen meines Arztes zu verstehen, da mir die Wörter durchsichtig werden. So konnte ich etwa kürzlich Cephalalgie als schlichtes "Kopfweh" identifizieren. Auch Ärzten - ob sie nun Griechisch gelernt haben oder nicht ist das Wort vermutlich nicht nur bekannt, sondern auch durchsichtig. Einem gewissen Personenkreis könnte das Wort mit seiner richtigen Bedeutung und Anwendung bekannt sein, ohne daß die Bestandteile analysiert werden können; für diese Personen wäre es ein undurchsichtiges Wort. Häufig wird es bei Wörtern dieser Art allerdings vorkommen, daß sie den Benützern bekannt und durchsichtig, den meisten Leuten aber ebenso unbekannt wie undurchsichtig sind. Dies bedeutet, daß die Verwendung eines Wortes und seine mögliche Durchsich-

24) Wir bleiben dabei auf der Ebene der Synchronie und schließen den historischen Sprachwissenschaftler, der die früheren sprachstufen kennt, aus. Allerdings: selbst Sprachwissenschaftler sind Menschen und damit Sprachbenutzer.

25) Vgl. M. Wandruszka, Die Mehrsprachigkeit des Menschen, München-Zürich 1979 . 
tigkeit in den Fachsprachen weitgehend parallel gehen; das Problem der eingeschränkten Durchsichtigkeit stellt sich hier vor allem dann, wenn fachsprachliche wörter aus ihrer Fachsprache in den Bereich der Allgemeinsprache geraten (so etwa in den letzten Jahren dt.Thermostat).

\section{Ergebnisse}

Unter den Einschränkungen der Wortdurchsichtigkeit sind Lexikalisierung im Einzelfall und prinzipielle Teildurchsichtigkeit mancher Wortbildungsprogramme zu unterscheiden. Die Gründe für Lexikalisierung können in der Realität der Welt, im lautlichen und im semantischen Bereich Iiegen. Im Vorgang der Lexikalisierung entfernen sich jeweils die Bestant teile eines mehrmorphemischen Wortes von entsprechenden Morphemen in freier Verwendung. Es gibt Wortbildungsprogramme, bei denen die Lexikalisierung sogar zu den konstitutiven Merkmalen gehört.

Bei der prinzipiellen Teildurchsichtigkeit von Wortbildungsprogrammen besteht dagegen keine Distanz der beteiligten Morpheme innerhalb und außerhalb des mehrmorphemischen Wortes; eine solche Distanz (Lexikalisierung) kann allenfalls zusätzlich hinzukommen. Die Definition von Wörtern, die nach diesen Programmen gebildet wurden, enthät aber mit Notwendigkeit lexikalische Elemente, welche bei einer Analyse allein aufgrund der Bestandteile und der Bildungsregeln nicht auffindbar sind.

Die getroffenen Unterscheidungen werden von der Tatsache überlagert, daß Durchsichtigkeit immer erst von den Sprachbenutzern realisiert wird und damit abhängig ist von deren graduell verschiedenen Fähigkeiten zur Sprachreflexion. 
Semantika skuša ločiti tako imenovane 'prozorme', motivirane besede od tistih, katerih vir je nejasen. Razpravljanje o prozornosti je s sinhronega stališča za večino besed nesmiselno, če sprejmemo kot izhodišče Saussurovo temeljno idejo o arbitrarnosti jezikovnega znaka, da namreč ni logične povezanosti med označenim in označujočim; a Saussure sam je izvzel iz te arbitrarnosti onomatopoetične besede in pa seveda tiste izraze, ki so sekundarno motivirani. Avtor se v svoji študiji omejuje na tiste 'prozorme' besede, $k i$ so sestavljene vsaj iz dveh elementov in je mogoče njih prozornost analizirati, ko analiziramo vsakega od delov in pa način gradnje. Popolno razumevanje motiviranosti pa je vendar omejeno z leksikalizacijo, s posebnimi zahtevami gradnje sintagme in končno $z$ dejstvi, ki so odvisna od jezikovnega uporabnika.

Dovolj znano je dejstvo, da jeziki med seboj ne morejo biti skladni: nemščina kot izrazit jezik kompozicije pozna za pojem 'jablana, sestavljenko Apfelbaum, romanski jeziki pa se zatekajo $k$ derivaciji kot fr. pommier, severmo-ital. pomaro, ali pa celo uporab1jajo gramatikalne morfeme it. melo, šp. manzano. Seveda se prozornost lahko izgubi: lat. RECIPERE je še vezano na CAPERE, fr. recevoir pa ne, ker se latinski enostavni glagol $v$ francoščini ne nadaljuje. Razstavljanje je seveda teoretično mogoče: prê- v fr. prelude ni motivirano, ker $v$ francoščini nekaj kot ${ }^{*}$ lude ne eksistira, tudi če je jasno, da sta v lat. PRELUDIUM oba dela motivirana, da je torej sintagma 'prozorna'.

Avtor misli, da omejuje prozornost besed leksikalizacija; oba dela sestavljenke se pomensko omejita in sestavljenka lahko pomeni nekaj povsem novega: $v$ modermi francoščini après-ski ne pomeni 'čas, ki ga prebijemo po smučanju', kot bi upravičeno sklepali po après-midi, ali po it. dopopranzo, dopolavoro, ampak 
pomeni sicer zimsko in toplo, a vendar lažjo obutev, ki si jo smučar natakne po koncu smučanja. Do napačnega sklepanja nas lahlo privedejo morfemi, ki se zdijo močno specializirani: morfem -iste najdemo $v$ seriji pianiste, violiniste; a visagiste ni slikar portretov.

In končno, prozomosti se govoreči velikokrat niti ne zaveda, saj je spoznanje motiviranosti odvisno od njegove sposobnosti za analizo, za razmišljanje o jezikovnih pojavih. Avtor opozarja tudi na izraze, privzete iz tujih jezikov: v evropskih jezikih je, na primer, čedalje več latinizmov in grecizmov, ker jih zahteva tehnični jezik, zanesljivo pa te izraze razume čedalje manjše število ljudi. In kadar gre za tujke z dvema prvinama, avtor si je za zgled izbral fr. carnivore, ovipare, oviforme, ovorde, ovipositeur, ignifuge, ignivome, je prav verjetno, da so ozkemu krogu uporabnikov sicer pomensko razumljive, da pa niso več razumljive $\mathrm{v}$ svojih dveh delih. 